\section{P140 (continued)}

age was 49 years. Participants were predominantly female (84\%), Hispanic or Latino (62\%) and Black (25\%). Thirty-four percent of participants reported a diagnosis of hypertension and $21 \%$ reported a diabetes diagnosis. Over $\$ 125,000$ in incentives have been distributed through the program, $70 \%$ of which have been redeemed.

Conclusions: The majority of incentives distributed are redeemed and we anticipate the program will reach the intended audience.

Funding: USDA

\section{P141 Time to Cook! Developing a Conceptual Framework to Guide Healthy Eating Education in Time-Scarce Lifestyles}

SuzannePiscopo,PhD, R.Nutr, suzanne.piscopo@um.edu. mt, University of Malta, Rm. 324, Faculty of Education, Msida, Tal-Qroqq, Malta, MSD2080

Background: Time is often indicated as a main barrier to cooking from scratch and to healthy eating. This has implications for health outcomes and nutrition education.

Objective: This study reviewed existing literature on the perception of time as a barrier to home cooking; the time link with cooking skills and practices; and inclusion of time in food, nutrition, and cooking education in different settings and through different media. The goal was to generate a conceptual framework to help inform healthy eating education considering time-scarce lifestyles.

Study Design, Setting, Participants: A narrative literature review was conducted involving searches in Pub Med and Google Scholar using the keywords time, food preparation, cooking, and health, and by consulting other grey literature. Years covered were 2010-2020.

Measurable Outcome/Analysis: One hundred and two studies were reviewed and thematic analysis was used to elicit main time-related factors which directly or indirectly were involved in food choices, preparation and consumption, and food, nutrition, and cooking skills education.

Results: Key emergent themes included general time scarcity and consumption of low-nutritive quality food (eg eating more highly-processed, nutrient-poor foods through using convenience foods or eating fast food meals); duration of food preparation and consumption of specific foods (eg vegetables, fruit, cholesterol-rich foods); diversity of vehicles to successfully promote and aid home cooking efficiency (eg community courses, TV programs, mobile apps, video messaging); culinary nutrition education for health professionals to help patients overcome time barriers to healthier eating; creative healthy snack and meal inspiration; and practical skills to plan and prepare healthy meals ahead of time.

Conclusions: A conceptual framework based on evidence form the literature shows how time availability and usage have implications for diet quality, and how time can be addressed in education for healthy eating. Further research could test the conceptual framework in aiding development of practical nutrition education to suit contemporary time-scarce lifestyles.

Funding: None.

\section{P142 Vegetable Intake, Preferences, and Variety in Michigan Gardeners Over a Single Gardening Season}

Ghaida Batarseh, BS, Wayne State University; Katherine Alaimo, PhD, Michigan State University; Sarah Comstock, $P h D$, Michigan State University; Alyssa Beavers, $P h D, R D$, beavers.alyssa@wayne.edu, Wayne State University, 3225 Science Hall, 410 W Warren, Detroit, MI, 48202

Background: Gardening is associated with health and health behaviors, including vegetable intake. Qualitative research has indicated that gardening may increase preferences for vegetables, which may contribute to higher vegetable intake frequency found in gardeners, but quantitative research examining vegetable preferences of gardeners is scarce.

Objective: The objective of this pilot study was to examine vegetable preferences, variety, and intake consumed before gardening and during peak harvest time. This data was collected as part of a larger study examining the relationship between gardening and the composition of the gut microbiome.

Study Design, Setting, Participants: Participants were adults enrolled in a gardener support program that serves both home and community gardeners. Thirty-two percent of participants had no previous gardening experience, $36 \%$ had $1-5$ years of gardening experience, and $32 \%$ had 5 or more years of experience. Twenty-nine participants enrolled in the study and completed a baseline survey prior to beginning gardening and 25 of these completed a follow-up survey at the peak of garden harvest.

Measurable Outcome/Analysis: Surveys assessed vegetable intake frequency, preferences for 20 different vegetables, and number of the 20 different vegetables eaten in the past month (variety). Paired, 2-tailed t-tests were used to compare pre-gardening and harvest time survey data. T-tests were considered significant at $P<.05$.

Results: Preferences for vegetables were initially high and did not increase from baseline to harvest time. Vegetable consumption frequency in times per day also did not significantly change from baseline to harvest time. Vegetable intake variety significantly increased from $12.1(\mathrm{SD} \pm 2.9)$ at baseline to $13.6(\mathrm{SD} \pm 3.1)$ at harvest time $(P=.026)$.

Conclusions: In this study, we found gardeners consumed a greater variety of vegetables during harvest time than before gardening. Previous research has found that consuming a high variety of plant-based foods has a positive influence on gut microbiome diversity. This has relevance to the 